\title{
Study on Lignite-Blended Burning Technology in the 1025t/h Bituminous Boiler
}

\author{
Jinfeng MA ${ }^{1}$, Jingxing WU ${ }^{2}$, Tianshu ZHOU ${ }^{2}$, Jie LENG ${ }^{2}$, Chong JIANG ${ }^{2}$ \\ ${ }^{1}$ Shenyang Engineering Institute, Shenyang, China \\ ${ }^{2}$ Northeast Electric Power Research Institute Co., Ltd., Shenyang, China \\ Email: majf69@yahoo.com.cn
}

\begin{abstract}
Due to a serious shortage of the coal in Tonghua, a retrofit solution of mixing warm flue gas extracted from reversing chamber into the coal pulverizing system and cold air into the hot air coal pulverizing system is proposed so as to reduce oxygen content. At the end of the pulverizing system and medium temperature of the conveying system, dual-channel combustion burner is transformed into horizontal bias combustion burner. The measurement results show that $50 \%$ ratio of lignite blended in the $1025 \mathrm{t} / \mathrm{h}$ bituminous boiler is feasibility. It is also an important technology to reduce $\mathrm{NO}_{\mathrm{x}}$ pollutant emission.
\end{abstract}

Keywords: coal pulverizing system, blended coal burning, lignite, warm flue gas, explosion prevention, drying capacity

\section{1. 引 言}

爲拓寬燃煤範圍，國内許多研究單位開展了燃煤 掺燒的研究工作 ${ }^{[-3]}$ 。爲實現在中儲式制粉系統烟煤鍋 爐中大比例掺燒褐煤，文獻[4,5]采用在鍋爐轉向室抽 取中温爐烟掺人制粉系統的改造技術，在 $200 \mathrm{MW}$ 機 組烟煤鍋爐上成功實現了大比例掺燒褐煤。

目前通化地區煤源供應嚴重不足, 渾江發電責任 有限公司 $2 \times 1025 \mathrm{t} / \mathrm{h}$ 機組鍋爐原設計煤種屬于劣質烟 煤, 揮發分和熱值均較低, 如果直接摻燒褐煤, 制粉 系統爆炸和燃燒器燒損問題最爲突出。爲最大限度摻 燒褐煤，本文采取在轉向室抽吸中温爐烟摻人制粉系 統、熱風送粉系統中加入調温風的改造方案, 以降低 制粉系統末端氧量以及送粉系統介質的温度，提高制 粉系統防爆和幹燥能力; 將雙通道煤粉燃燒器改造爲 水平濃淡燃燒器, 保護燃燒器不被燒損, 并進行了改 造試驗研究。

\section{2. 設備概况}

渾江發電責任有限公司 1 號鍋爐爲亞臨界壓力自 然循環固態排渣爐, 型號爲 SG-1025/17.5-M889。采 用中儲式熱風送粉制粉系統，配置 4 臺鋼球磨煤機。 采用四角切圓燃燒方式, 雙通道煤粉燃燒器布置有 4 層，其中 3 層設有大油槍。
爐膛寬度爲 $12800 \mathrm{~mm}$ ，深度爲 $11890 \mathrm{~mm}$, 爐頂 管中心綫標高 $58000 \mathrm{~mm}$ 。過熱器由包墙過熱器、低温 過熱器、分隔屏、後屏及高温過熱器組成。再熱器由 低温再熱器和高温再熱器組成。後烟井爲并聯雙烟道, 後烟井前部爲低温再熱器烟道, 後烟井後部爲低温過 熱器烟道。在低温再熱器和低温過熱器的烟道下方布 置有省煤器受熱面。尾部堅井烟道中交錯布置着上級 省煤器、上級空氣預熱器、下級省煤器和下級空氣預 熱器。過熱蒸汽調温采用 I 、II 級噴水减温方式, 再 熱器的調温采用烟氣擋板調温方式。

\section{3. 改造方案}

\section{1 制粉系統改造方案}

在中儲式制粉系統中增加中温爐烟系統，送粉系 統中加人由送風機出口引人的冷風作爲調温風, 如圖 1 所示。中温爐烟管分左右 2 路引至爐前，再分 2 路 進人 4 臺磨煤機，管材爲 $12 \mathrm{Cr} 1 \mathrm{MoV}$ 。

根據相關試驗結果, 當制粉系統末端終端氧量低 于 16\%時, 任何條件下霍林河褐煤都不發生爆炸。煤 粉水分的選取直接影響制粉系統幹燥能力, 參考其它 電廠掺燒褐煤的試驗數據，將煤粉水分選取爲 $6 \%$, 送粉系統計算時選取爲 5\%。設計煤質與掺燒 50\%褐 煤的混配煤質特性示于表 1, 中温爐烟系統及送粉系 


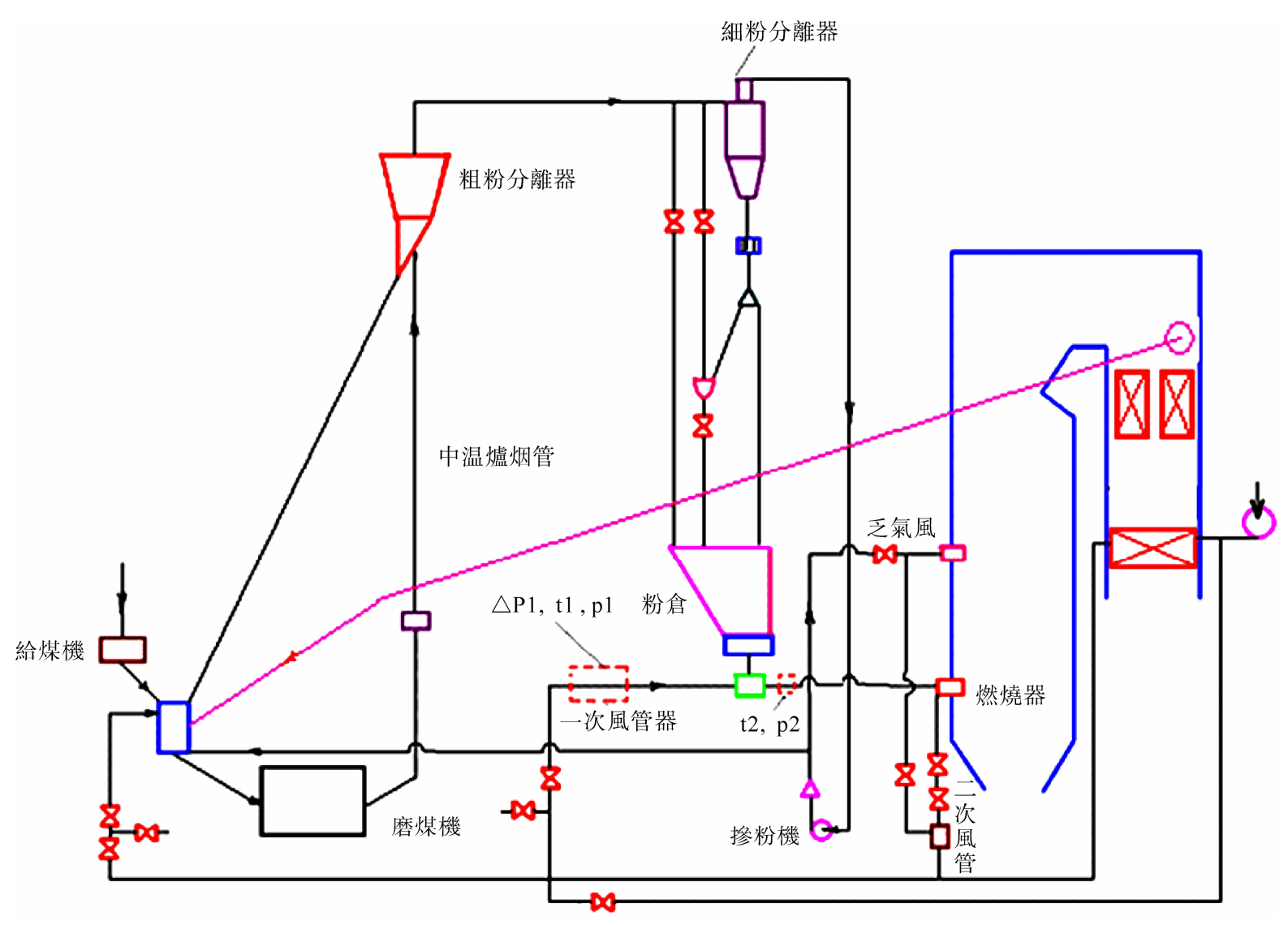

圖1. 中温爐烟系統示意圖

Figure 1. Piping arrangement diagram of warm flue gas system

表 1. 煤質主要特性表

Table 1. main characteristics of the coal

\begin{tabular}{cccc}
\hline 名 稱 & $\begin{array}{c}\text { 設 計 } \\
\text { 烟 煤 }\end{array}$ & $\begin{array}{c}\text { 霍林河 } \\
\text { 褐 煤 }\end{array}$ & $\begin{array}{c}\text { 混 配 } \\
\text { 煤 種 }\end{array}$ \\
\hline $\mathrm{C}_{\text {ar }} / \%$ & 43.38 & 35.75 & 39.67 \\
$\mathrm{H}_{\text {arl }} / \%$ & 2.49 & 2.63 & 2.6 \\
$\mathrm{O}_{\mathrm{ar}} / \%$ & 5.3 & 9.67 & 7.76 \\
$\mathrm{~N}_{\text {arr }} / \%$ & 0.7 & 0.71 & 0.71 \\
$\mathrm{~S}_{\mathrm{ar}} / \%$ & 0.41 & 0.34 & 0.37 \\
$\mathrm{M}_{\text {ar }} / \%$ & 6.02 & 29.29 & 17.66 \\
$\mathrm{~A}_{\text {ar }} / \%$ & 41.7 & 21.61 & 31.23 \\
$\mathrm{~V}_{\text {daf }} / \%$ & 27.38 & 45.9 & 38.91 \\
$\mathrm{Q}_{\text {ar, net }} / \mathrm{kg} \cdot \mathrm{kg}^{-1}$ & 16200 & 12780 & 14530 \\
\hline
\end{tabular}

統部分理論計算結果示于表 2 , 鍋爐熱力計算部分結 果示于表 3 。

由表 3 計算結果可知, 在 BMCR 條件下鍋爐計算 效率將會降低 $2.24 \%$ 。而在實際運行時, 制粉系統滿
負荷下通常衹采用 2 3 套運行, 很少采用 4 套同時運 行, 這樣送粉系統所用冷風量會减少; 掺燒褐煤後混 煤燃盡率將會提高, 機械未完全燃燒損失將會减少, 實際運行中鍋爐效率將會進一步提高。 
表 2.中温爐烟系統及送粉系統計算結果

Table 2. Calculation results of warm flue gas system and conveying system

\begin{tabular}{lc}
\hline \multicolumn{1}{c}{ 名 稱 } & 計算結果 \\
\hline 烟煤、褐煤比例 & \\
煤粉細度 $\left(\mathrm{R}_{90}\right) / \%$ & $1: 1$ \\
抽取點過量空氣系數 & 16 \\
一次風率 $/ \%$ & 1.27 \\
空氣幹燥基水分 $/ \%$ & 23 \\
煤粉水分 $/ \%$ & 5 \\
實際燃料消耗量 $/ \mathrm{t} \cdot \mathrm{h}^{-1}$ & 5 \\
理論幹空氣量 $/ \mathrm{Nm}^{3} \cdot \mathrm{h}^{-1}$ & 205 \\
磨煤機最佳通風量 $/ \mathrm{m}^{3} \cdot \mathrm{h}^{-1}$ & 3.97 \\
中温爐烟占總烟氣量的質量分額 $/ \%$ & 145779 \\
制粉系統末端氧容積份額/\% & 5.4 \\
系統阻力 $/ \mathrm{Pa}$ & 13.9 \\
送粉系統氣粉混合物温度 $/{ }^{\circ} \mathrm{C}$ & 659 \\
& 90 \\
\hline
\end{tabular}

表 3. 熱力計算結果

Table 3. Thermal calculation results

\begin{tabular}{|c|c|c|c|c|}
\hline 項 & $\begin{array}{c}\text { 四臺磨投運 } \\
\text { 設計煤種 } \\
\text { BMCR }\end{array}$ & $\begin{array}{c}\text { 四臺磨投運 } \\
\text { 摻燒 } 50 \% \text { 褐煤 } \\
\text { BMCR }\end{array}$ & $\begin{array}{c}\text { 磨煤機全停 } \\
\text { 摻燒 } 50 \% \text { 褐煤 } \\
\text { BMCR }\end{array}$ & $\begin{array}{c}\text { 三臺磨投運 } \\
\text { 摻燒 } 50 \% \text { 褐煤 } \\
\text { ECR }\end{array}$ \\
\hline 空氣預熱器人口烟氣量 $/ \mathrm{t} \cdot \mathrm{h}^{-1}$ & 1339.2 & 1451.5 & 1406.3 & 1312.4 \\
\hline 排烟温度 ( 未修正 ) / ${ }^{\circ} \mathrm{C}$ & 149 & 180 & 141 & 171 \\
\hline 排烟温度（修正 ）/ ${ }^{\circ} \mathrm{C}$ & 139 & 171 & 133 & 161 \\
\hline 低温過熱器進口烟温 $/{ }^{\circ} \mathrm{C}$ & 648 & 660 & 652 & 643 \\
\hline 鍋爐熱效率/\% & 90.80 & 88.56 & 90.96 & 89.08 \\
\hline 計算燃料量/ $\mathrm{t} \cdot \mathrm{h}^{-1}$ & 178.5 & 202.5 & 196.2 & 183.1 \\
\hline 實際燃料量/ t · $\mathrm{h}^{-1}$ & 182.9 & 207.5 & 201.0 & 187.6 \\
\hline
\end{tabular}

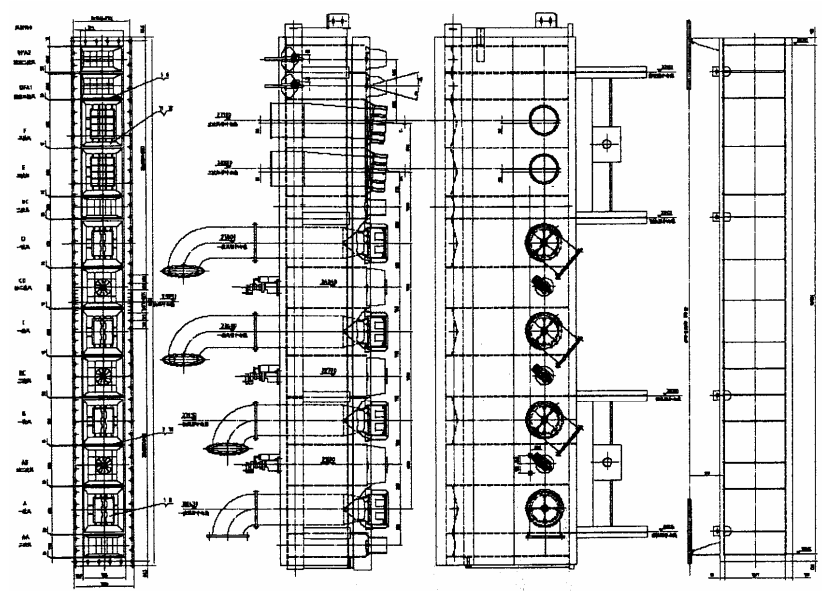

圖 2. 煤粉燃燒設備示意圖

Figure 2. Diagram of combustion burner 


\section{2. 燃燒器改造方案}

燃燒器原設計采用大速差雙通道煤粉燃燒器, 一 次風噴口布置相對集中。掺燒褐煤的混配煤質着火性 能和燃盡性能提高, 同時結焦傾向也增加, 因此將燃 燒器改爲均等配風布置。燃燒器四角的安裝角度不變, 燃燒器水冷套不變, 燃燒器各層風室重新分配 ( 調整 風室高度), 調整全部一、三次風標高, 調整二層剛性 梁標高等。同時改變設計參數, 如提高一次風率和風 速等。燃燒設備示意圖如圖 2 所示。

該燃燒器設計特點如下:

（1）采用中温爐烟+熱風+冷風+再循環送粉系統。

(2) 一次風設計采用成熟的WR水平濃淡燃燒器。 一次風噴嘴中裝有 $\mathrm{V}$ 型鈍體, 在 $\mathrm{V}$ 型鈍體前方形成穩 定的回流區卷吸高温烟氣, 起到穩燃作用。燃燒器的 一次風噴嘴采用均等布置, 三次風噴嘴集中布置。
(3) 二次風設計按 “加强混合, 分級然燒” 的原 則進行, 既有利于着火、穩燃和燃燼, 又减少 $\mathrm{NO}_{\mathrm{x}}$ 的 生成量。在燃燒器頂部設置 2 層燃燼風, 爲手動擺動 噴嘴, 其餘噴嘴均爲固定噴嘴。射流旋轉方向與其餘 的一、二次風射流旋轉方向相反, 以减少鍋爐水平烟 道左右兩側的烟温偏差。

\section{4. 中温爐烟系統投運試驗}

\section{1. 抽熱爐烟試驗結果}

爲驗證本次改造的實際效果, 現場設備安裝完成 後, 進行了磨煤機額定出力下抽吸中温爐烟試驗。試 驗過程中爐烟調節擋板保持全開, 通過改變磨煤機人 口負壓調整中温爐烟量以及制粉系統末端氧量。磨煤 機人口負壓與制粉系統末端含氧量、抽取烟氣量的關 系曲綫如圖 3、圖 4 所示。

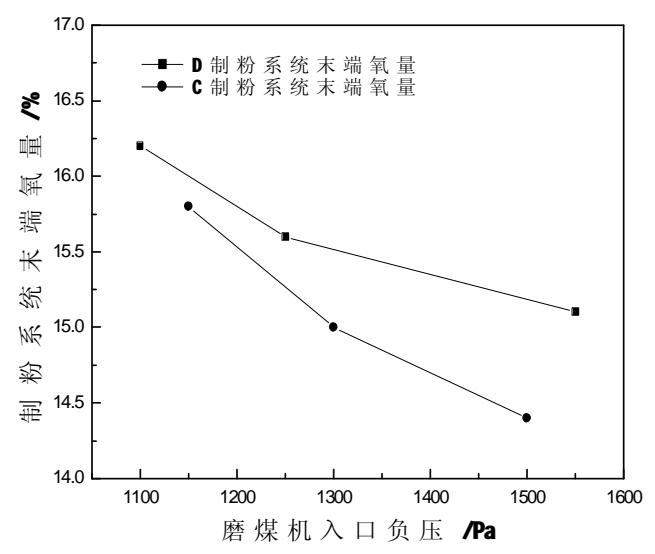

圖 3. 磨煤機人口壓力與制粉系統末端含氧量關系圖

Figure 3. Interrelation between oxygen content at the end

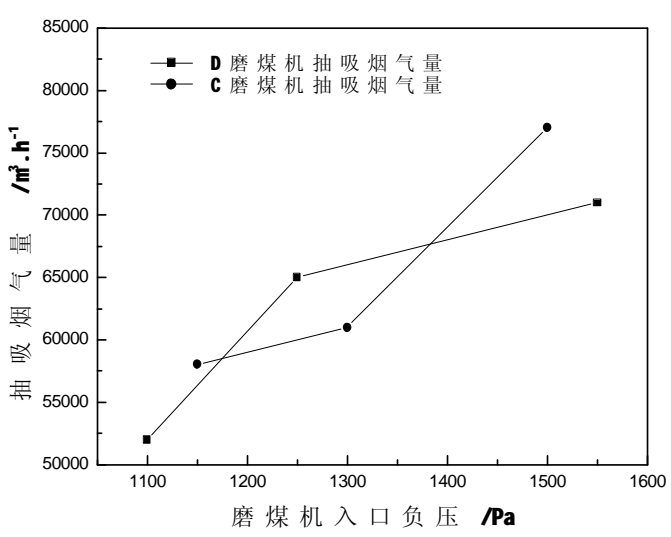

圖 4. 磨煤機人口壓力與抽取烟氣量關系圖

Figure 4. Interrelation between flue gas flow rate 
表 4. NOx 排放量測試結果

Table 4. The NOx emissions results

\begin{tabular}{|c|c|c|c|c|}
\hline \multirow{3}{*}{ 名 稱 } & \multicolumn{4}{|c|}{ 測 試 數 據 } \\
\hline & \multicolumn{2}{|c|}{$33 \%$ 比例褐煤 } & \multicolumn{2}{|c|}{$50 \%$ 比例褐煤 } \\
\hline & 不投運 & 投運 & 不投運 & 投運 \\
\hline 機組負荷/ MW & 209 & 211 & 213 & 211 \\
\hline 空氣預熱器出口烟氣含氧量/\% & 6.5 & 6.2 & 6.8 & 6.5 \\
\hline 空氣預熱器出口 NOx 的體積濃度/ppm & 199.4 & 183 & 227 & 195.3 \\
\hline 折算後的預熱器出口 NOx 的體積濃度/ ppm & 205.6 & 186 & 239.8 & 202.2 \\
\hline 空氣預熱器出口 NOx 的質量濃度/ $\mathrm{mg} \cdot \mathrm{Nm}^{-3}$ & 421.5 & 381.2 & 491.6 & 414.5 \\
\hline 降低幅度/\% & \multicolumn{2}{|c|}{9.6} & \multicolumn{2}{|c|}{15.7} \\
\hline 中温爐烟再循環率/\% & \multicolumn{2}{|c|}{6} & \multicolumn{2}{|c|}{7} \\
\hline
\end{tabular}

圖 3 的試驗數據表明, 制粉系統末端含氧量隨着 磨煤機人口負壓的增加呈現遞减趨勢。C、D 兩套制 粉系統正常運行時人口負壓經常保持在 $1300 \mathrm{~Pa}$ 左右, 末端含氧量低于 $16 \%$, 滿足了制粉系統的防爆要求。 圖 4 的試驗數據表明, 隨着磨煤機人口負壓的提高, 抽取的中温爐烟量逐漸增加, 磨煤機出口温度升高, 制粉系統幹燥出力增强, 三個試驗工况下磨煤機出口 温度均能控制在 $60^{\circ} \mathrm{C}$ 以上。可見, 改造後鍋爐的防爆 和幹燥能力提高。

\section{2. 低 $\mathrm{NO}_{\mathrm{x}}$ 排放試驗結果}

燃煤電站低 $\mathrm{NO}_{x}$ 排放技術主要包括空氣分級燃燒 技術、燃料再燃燒技術、烟氣再循環以及烟氣處理 SNCR 和 SCR 方法 ${ }^{[6-10]}$ 。在鍋爐轉向室抽取中温爐烟 掺人制粉系統實現了烟氣再循環利用, 將雙通道煤粉 燃燒器改造爲水平濃淡燃燒器實現了分級燃燒。

本文在渾江發電責任有限公司 1 號鍋爐上進行了 改造後 $\mathrm{NO}_{\mathrm{X}}$ 排放試驗, 測點設在空氣預熱器出口處。 表 4 爲掺燒 33\% 和 50\%比例褐煤時 $\mathrm{NO}_{\mathrm{x}}$ 排放測試結果。

表 4 中的測試結果表明, 改造後 $\mathrm{NO}_{\mathrm{x}}$ 排放質量濃 度均低于文獻[11]中第 3 時段規定的最高允許排放質 量濃度 $\left(450 \mathrm{mg} \cdot \mathrm{Nm}^{-3}\right)$ 。鍋爐掺燒 $33 \%$ 比例褐煤、兩 套中温爐烟系統投運前後 NOx 排放質量濃度分别爲 $421.5 \mathrm{mg} \cdot \mathrm{Nm}^{-3}$ 和 $381.2 \mathrm{mg} \cdot \mathrm{Nm}^{-3}$, 後者比前者降低了 $9.6 \%$ 。鍋爐掺燒 50\%比例褐煤、兩套中温爐烟系統投 運前後 NOx 排放質量濃度分别爲 $491.6 \mathrm{mg} \cdot \mathrm{Nm}^{-3}$ 和 $414.5 \mathrm{mg} \cdot \mathrm{Nm}^{-3}$, 後者比前者降低了 $15.7 \%$ 。這是由于 掺燒褐煤後, 由于混煤水分增加, 爐膛火焰温度有所
降低, 减少了熱力型 $\mathrm{NO}_{\mathrm{x}}$ 的生成; 中温爐烟作爲一次 風進人燃燒器區域後, 降低了燃燒器區域的氧氣濃度, 抑制煤粉燃燒中燃料型 $\mathrm{NO}_{\mathrm{x}}$ 的形成。同時, 雙通道煤 粉燃燒器改造爲水平濃淡燃燒器, 進一步减少了燃料 型 $\mathrm{NO}_{\mathrm{x}}$ 的形成, 從而减輕了對大氣環境的污染。

\section{5. 結 論}

(1) 在鍋爐轉向室抽取中温爐烟摻人制粉系統、 在送粉系統加人冷風作爲調温風的改造技術, 增强了 $1025 \mathrm{t} / \mathrm{h}$ 鍋爐中儲式制粉系統的防爆能力和幹燥能力; 雙通道煤粉燃燒器改造爲水平濃淡燃燒器, 解决了燃 燒器燒損問題, 達到了劣質烟煤鍋爐中大比例摻燒褐 煤的預期目標。

(2)由于水平濃淡燃燒技術與烟氣再循環利用的 雙重影響, 降低了 NOx 的排放量, 因此中儲式制粉系 統烟煤鍋爐掺燒褐煤的改造技術同樣是一種重要的减 排技術。

\section{REFERENCES}

[1] J. W. Shu, F. B. Meng, Q. L. Huang, et al., "The calculation and selection of drying agents in connection with the change of fuel for a boiler from brown coal to bituminous coal," Journal of Engineering for Thermal Energy and Power, Vol. 7, pp. 453-456, 2001.

[2] J. Guo, R. Jiang, and H. C. Zeng, "Formation of NOx during blended coals combustion process and it's computer simulation," Power System Engineering, Vol. 10, No. 2, pp. 55-60, 1999.

[3] Y. H. Li, H. W. Chen, J. Z. Liu, et al., "Numerical simulation of blending coals combustion of 800 MW boiler," 
Proceedings of the CSEE, Vol. 22, No. 6, pp. 101-104, 2002.

[4] J. X. Wu, H. G. Chen, J. F. Ma, et al., "Feasibility study on the hot flue gas mixture in coal pulverizing system of 200MW boiler," Electric Power, Vol. 39, No. 3, pp. 22-25, 2006.

[5] J. F. Ma, J. X. Wu, T. S. Zhou, et al., "Study on lignite blended burning technology in the bin and feeder coal pulverizing system," Journal of Power Engineering, Vol. 28, No. 1, pp. 14-18, 2008.

[6] S. Miyamae, T. Kiga, H. Ikebe, K. Makino, et al., "Low $\mathrm{NO}_{\mathrm{x}}$ pulverized coal combustion technology for large utility thermal power plant," Coal Combustion Science and Technology of Industrial and Utility Application, Hemisphere Publishing Corporation, New York, 1988.

[7] M. Berg and H. Bering, "Development of a low- $\mathrm{NO}_{\mathrm{x}}$ burner for pulverized- coal combustion and retrofitting of a full-scale power plant boiler," Proceedings of the $2^{\text {nd }}$
International Symposia on Coal Combustion, China Machine Press, Beijing, 1991.

[8] E. Troconi, L. Lietti, P. Forzatti, et al., "Experimental and theoretical investigation of the dynamics of the SCR$\mathrm{DeNO}_{\mathrm{x}}$ reaction, Chemistry Science, Vol. 51, No. 11, pp. 2965-2970, 1996.

[9] C. G. Yin, S. Cailat, and J. Harion, "Investigation of flow, combustion, heat-transfer and emission from a 609MW utility tangentially fired pulverized-coal boiler," Fuel, Vol. 81, No. 8, pp. 997-1006, 2002.

[10] M. Falcite, S. Pasini, and L. Tognotti, "Modeling paractical combustion systems and predicting $\mathrm{NO}_{\mathrm{x}}$ emission with an integrated CFD based approach," Computers and Chemical Engineering, Vol. 26, No. 9, pp. 1171-1183, 2002.

[11] Fossil-fuel Power Plant New Atmospheric Pollutant Emission Standard, GB13223-2003, Electric Power Press, Beijing, China, 2003. 